\title{
Perfil epidemiológico, clínico e de independência funcional de uma população idosa institucionalizada
}

\author{
Epidemiological, clinical and of functional independence profile of an institutionalized elderly population \\ Perfil epidemiológico, clínico e de independencia funcional de una \\ población de ancianos institucionalizados
}

\author{
Cristiane Rabelo Lisboa', Tânia Couto Machado Chianca" \\ ' Universidade Federal de Minas Gerais, Escola de Enfermagem. Belo Horizonte-MG, Brasil. \\ "Universidade Federal de Minas Gerais, Escola de Enfermagem, Programa de Pós Graduação em Enfermagem, \\ Núcleo de Estudos e Pesquisas sobre Sistematizar o Cuidar em Saúde e Enfermagem, e Núcleo de Estudos e \\ Pesquisas em Enfermagem Baseada em Evidências. Belo Horizonte-MG, Brasil.
}

\author{
Submissão: 25-01-2011 Aprovação: 19-07-2012
}

\begin{abstract}
RESUMO
Estudo descritivo transversal com objetivo de descrever características sócio-demográficas, clínicas e o nível de independência funcional de 97 idosos institucionalizados nas duas instituições de longa permanência de Itaúna-MG. Utilizou-se instrumento com variáveis sócio-demográficas e clínicas, além da Escala de Independência em Atividade da Vida Diária de Katz. Observou-se predomínio de idosas $(59 \%)$, média de idade de 77 anos, analfabetos $(55 \%)$, brancos $(67 \%)$, solteiros $(63 \%)$, sem filhos $(68 \%)$ e $30 \%$ que moravam sozinhos e tinham dificuldades para realizar atividades de vida diária, sendo este o motivo principal da institucionalização. A maioria dos idosos (72\%) apresentava doenças cardiovasculares; $80,4 \%$ usavam neurolépticos/psicotrópicos; $2,1 \%$ não usavam medicamentos e $23 \%$ eram independentes para realizar atividades de vida diária. Estes idosos demandam cuidados mais qualificados, justificados pela dependência, patologias, riscos de complicações e medicamentos utilizados.

Descritores: Instituição de Longa Permanência para Idosos; Saúde do Idoso; Envelhecimento da População.
\end{abstract}

\begin{abstract}
Descriptive and sectional study to describe socio-demographic, clinical and functional independence profile of 97 institutionalized elderly in two nursing homes of Itaúna-MG. A socio-demographic and clinical data and Independence Daily Living Activity of Katz instruments were used. It was found a predominance of elderly women (59\%), mean age of 77 , illiterate $(55 \%)$, white $(67 \%)$, single $(63 \%)$, without children $(68 \%)$ and $30 \%$ lived alone and had difficulty to perform daily living activities, which is the main reason for institutionalization. The majority of elderly people $(72 \%)$ had cardiovascular disease; $80.4 \%$ used neuroleptics/ psychotropic drugs, only $2.1 \%$ did not use drugs and $23 \%$ were independent to perform daily living activities. These seniors require more skilled care, justified by addiction, diseases, risk of complications and medications used. Key words: Homes for the Aged; Health of the Elderly; Demographic Aging.
\end{abstract}

\section{RESUMEN}

Estudio descriptivo de corte transversal, para describir perfil socio-demográfico, clínico y de independencia funcional de 97 ancianos institucionalizados en dos residencias de ancianos de Itaúna, Minas Gerais. Fueron utilizados instrumentos con dados socio-demográficos y clínicos y de Independencia en actividades de vida diaria de Katz. Se encontró un predominio de mujeres con edad avanzada (59\%), media de 77 años, analfabetos (55\%), blancos $(67 \%)$, solo $(63 \%)$ y sin hijos $(68 \%)$ y $30 \%$ tenía dificultad para realizar las actividades de vida diaria, razón principal para institucionalización. La mayoría de las personas de edad avanzada (72\%) tenían enfermedad cardiovascular, 80,4\% utilizan neurolépticos o drogas psicotrópicas y 2,1\% no usó drogas. $23 \%$ eran independientes para realizar las actividades de vida diaria. Estos adultos mayores requieren más atención especializada, justificado por la adicción, enfermedades, riesgo de complicaciones y medicamentos utilizados.

Palabras clave: Hogares para Ancianos, Salud del Anciano; Envejecimiento de la Población. 


\section{INTRODUÇÃO}

O envelhecimento populacional é uma realidade na maioria das sociedades atuais, decorrente de mudanças ocorridas em alguns indicadores de saúde, especialmente naqueles relacionados a quedas na fecundidade e na mortalidade, que aliados aos avanços tecnológicos e científicos, têm possibilitado um aumento na expectativa de vida ${ }^{(1)}$. Este é considerado um fenômeno mundial que, no Brasil, vem ocorrendo de maneira bastante acelerada ${ }^{(2)}$.

Estima-se que em 2050 o número de pessoas idosas no mundo poderá chegar a dois bilhões de indivíduos. Os números atuais mostram que uma em cada dez pessoas tem 60 anos de idade ou mais e, para 2050, a relação será, em média, de uma para cinco em todo o mundo. Por sua vez, nos países desenvolvidos, prevê-se que essa relação será de uma para três pessoas. A projeção do número de idosos com 100 anos ou mais de idade no ano de 2050 é de 2,2 milhões ${ }^{(2)}$. Atualmente em nosso país, a população idosa é de aproximadamente 18 milhões de pessoas ${ }^{(3)}$. Em Itaúna, município localizado na região Centro-Oeste de Minas Gerais, a população idosa é de 7.597 pessoas, correspondendo a aproximadamente 8,9\% da população total do município(4).

Estudo descritivo realizado em Belo Horizonte-MG, com dados secundários da Secretaria Municipal de Coordenação de Políticas Sociais, descreveu o perfil epidemiológico da população idosa na cidade e encontrou que, em 2000, os idosos representavam $9,1 \%$ do total da população residente na capital mineira (60,5\% mulheres e 39,4\% homens). Encontrou-se que 2,9\% dos idosos apresentavam algum tipo de deficiência física, $16,4 \%$ de deficiência auditiva, $23,5 \%$ de dificuldade para enxergar, $24,1 \%$ de dificuldade para caminhar ou subir escadas e que $3,8 \%$ apresentavam problemas mentais. Os autores concluíram que a parcela de pessoas idosas fragilizadas em Belo Horizonte era inferior à representada por aquelas com boas condições de saúde ${ }^{(5)}$.

A fragilização no processo de envelhecimento se constitui em uma síndrome de origem multidimensional envolvendo um conjunto de fatores biológicos, psicológicos e sociais que levam o idoso a um estado de maior vulnerabilidade e ao maior risco de declínio funcional, de sofrer quedas, hospitalização e morte ${ }^{(6)}$.

Os idosos que desfrutam de boas condições de saúde e financeiras não trazem preocupações para a família e tampouco para a sociedade. São bons consumidores, além de ajudarem os seus familiares. Entretanto, quando ficam doentes e dependentes, demandando gastos financeiros com doenças e cuidados, passam a interferir na vida dos familiares, tornando-se uma fonte de preocupação.

No contexto social onde se insere a nova configuração da família, o ritmo de vida imposto pelo mundo capitalista e as dificuldades de ordem financeira da maioria das famílias brasileiras têm gerado obstáculos para a manutenção do idoso em seu lar. Além disso, a geração que hoje é responsável por assistir os mais velhos, sobretudo as mulheres, não está mais tão disponível para a prestação dos cuidados como antes.

Sendo assim, em decorrência da intensificação no processo de envelhecimento populacional somada à diminuição gradativa na capacidade da família em prestar os cuidados necessários aos seus membros mais idosos, parece ocorrer um aumento na demanda das pessoas por instituições de longa permanência para idosos (ILPI), anteriormente denominadas como asilos.

A maioria dos idosos independentes permanece junto às famílias. Quando a demanda por cuidados é mais intensa e sobrecarrega os familiares, os idosos, algumas vezes, são institucionalizados. No Brasil, as próprias pessoas que compõem as famílias assistem os idosos no maior período de tempo e tão bem quanto possível. Quando ocorre a institucionalização, os familiares dos idosos alegam ter chegado ao limite da capacidade de cuidar desses seus parentes ${ }^{(7)}$.

A demanda por cuidados advém das várias alterações fisiológicas que ocorrem com a senescência, com velocidades variáveis e mais ou menos acentuadas, de acordo com as características pessoais de cada idoso. A principal alteração é a diminuição progressiva na capacidade funcional, definida como competência para executar atividades que lhes permitem cuidar de si próprios e viver independentemente em seu meio $^{(8)}$.

A independência e a autonomia nas atividades da vida diária (AVDs) estão relacionadas ao funcionamento integrado de quatro grandes funções: cognição, humor, mobilidade e comunicação. Quando estas funções estão comprometidas, direta ou indiretamente, de forma isolada ou associada, em consequência de uma ou mais doenças, pode-se verificar um prejuízo na realização das $\mathrm{AVDs} \mathrm{s}^{(9)}$.

É sabido que o envelhecimento aumenta o risco de doenças crônicas não-transmissíveis que podem afetar a funcionalidade das pessoas levando-as a uma situação de dependência para o desempenho de AVDs, como banhar-se, vestir-se, alimentar-se, transferir-se da cama para a cadeira, usar o sanitário e manter a continência urinária, anal ou ambas. Assim, os idosos requerem cuidados pessoais em tempo integral. Essa dependência tende a aumentar em cerca de $5 \%$ na faixa etária de 60 anos para cerca de 50\% entre os idosos com 90 anos ou mais ${ }^{(6)}$.

Um dos instrumentos mais antigos e mais citados na literatura nacional e internacional para avaliação da capacidade funcional é o Índice de Independência em Atividade da Vida Diária (EIAVD) ${ }^{(10)}$. Este instrumento foi desenvolvido e publicado em 1963 por Sidney Katz e sua equipe, com o objetivo de fazer uma avaliação dos resultados de tratamentos em idosos bem como fornecer uma predição do prognóstico nos doentes crônicos $^{(11)}$. Em 2008, foi publicada uma adaptação transcultural para o português da Escala de Independência em Atividades da Vida Diária, usualmente denominada como Escala de Katz ${ }^{(11)}$. É um instrumento de medida das AVDs hierarquicamente relacionadas e está organizado para mensurar a independência no desempenho de seis funções consideradas básicas e biopsicossocialmente integradas: banhar-se, vestir-se, ir ao banheiro, transferir-se da cama para a cadeira e vice-versa, ser continente e alimentar-se ${ }^{(10)}$. Cada função possui três alternativas categorizadas desde independente a dependente $^{(11)}$. 
A independência significa que a função é realizada sem supervisão. Esta avaliação é feita de acordo com a situação real e não com a habilidade de realizar a tarefa sem auxílio de terceiros. Então, se o sujeito tem capacidade de executar uma função, mas se nega a cumpri-la, ou se as condições ambientais são desfavoráveis, considera-se que ele não a realiza. Assim, um idoso que necessita de que um parente esteja presente dentro do banheiro, por exemplo, para que possa tomar banho é considerado dependente, apesar de ser capaz de realizar $\mathrm{o}$ ato sem o auxílio direto(11-12).

O idoso muitas vezes é institucionalizado apresentando comprometimento em sua capacidade funcional, em condições de grande fragilidade e necessitando de cuidados de enfermagem. Além disso, não se conhece o perfil (epidemiológico, clínico e de independência funcional) da população idosa institucionalizada em Itaúna-MG. Assim, realizou-se este estudo para descrever o perfil dos idosos institucionalizados neste município. Conhecer esta população poderá fornecer subsídios que auxiliarão na formulação e implementação de políticas públicas que possam melhor atender às reais necessidades dos idosos, assim como auxiliar na alocação de recursos que possibilitem uma assistência mais adequada e que possam contribuir para a melhoria da qualidade de vida desses idosos.

\section{MATERIAL E MÉTODOS}

Trata-se de um estudo descritivo transversal, desenvolvido nas duas instituições de longa permanência para idosos (ILPI) existentes no município de Itaúna-MG. Ambas são de caráter filantrópico, intituladas Instituição A e Instituição B, preservando o sigilo quanto aos seus nomes. A instituição $A$ tem capacidade para 75 idosos e a instituição B para 45 idosos. $\mathrm{Na}$ instituição $\mathrm{A}$, a assistência era prestada por uma médica generalista, um psiquiatra, uma psicóloga, uma assistente social, uma enfermeira, duas auxiliares de enfermagem e 14 cuidadores leigos, que não possuíam formação e capacitação técnica, sendo que 11 trabalhadores se revezavam em plantões de 12 por 24 horas no turno diurno e 3 no noturno. A instituição $B$ não possuía enfermeiro e a assistência era prestada por três técnicas de enfermagem e sete cuidadores leigos. A assistência de enfermagem nas duas instituições não era sistematizada.

A coleta dos dados foi realizada no período de 30 de abril a 30 de julho de 2009. A população do estudo foi constituída por idosos com 60 anos ou mais, residentes nestas instituições durante o período da coleta de dados e que concordaram em participar do estudo. Assim, a amostra final constituiu-se de 70 idosos na instituição A e 27 idosos na instituição B, perfazendo um total de 97 pessoas idosas.

Para a coleta de dados, utilizou-se um instrumento que abordava variáveis de identificação do idoso, variáveis sócio-demográficas, condições clínicas e variáveis referentes à avaliação funcional estabelecida segundo a Escala de Katz. As informações foram coletadas com cada idoso, com seus familiares, em seus prontuários ou com cuidadores e profissionais de saúde das instituições.
A avaliação da capacidade funcional, conforme a Escala de Katz, foi realizada de acordo com os diferentes graus de independência funcional estabelecidos para cada função, sendo os índices de 0 a 6: 0 - independente em todas as seis funções; 1 - independente em cinco funções e dependente em uma função; 2 - independente em quatro funções e dependente em duas; 3 - independente em três funções e dependente em três; 4 - independente em duas funções e dependente em quatro; 5 - independente em uma função e dependente em cinco funções; 6 - dependente em todas as seis funções ${ }^{(11)}$.

$\mathrm{O}$ instrumento foi aplicado por quatro enfermeiras, treinadas especificamente para a colaboração na aquisição dos dados, sendo uma delas pesquisadora deste estudo.

O estudo foi aprovado pelo Comitê de Ética em Pesquisa da Universidade Federal de Minas Gerais (Parecer COEP - ETIC 267/09). Para participar, cada idoso e seu responsável foram convidados e receberam orientações sobre o estudo. Aqueles que concordaram em participar assinaram o Termo de Consentimento Livre e Esclarecido - TCLE, conforme preconiza a Resolução N¹96/96 do Conselho Nacional de Saúde.

Todos os dados coletados foram digitados em uma planilha do Statistic Package for Social Sciences (SPSS), versão 16.0 e foi realizado o procedimento de dupla digitação para avaliação de consistência do banco de dados. Utilizou-se análise descritiva com apresentação de frequências simples e percentuais, média, desvio-padrão, valores máximos e valores mínimos.

\section{RESULTADOS}

Buscou-se conhecer as características dos idosos institucionalizados, avaliando variáveis sócio-demográficas, clínicas e o nível de independência funcional apresentado por eles.

Observou-se um predomínio de idosos do sexo feminino (59\%). A idade dos idosos variou entre 61 e 94 anos, com média de 77 anos e desvio padrão de 8,5 anos, sendo que a maior parte deles estava com 80 ou mais anos $(40 \%)$. Em relação ao grau de escolaridade, $55 \%$ dos idosos institucionalizados eram analfabetos, sendo $28 \%$ homens e $72 \%$ mulheres. Os idosos de cor ou raça branca eram maioria, correspondendo a $67 \%$ da amostra estudada.

Em relação ao estado civil e número de filhos, encontrou-se um predomínio de idosos solteiros (63\%) e sem filhos (68\%). Quanto ao local de procedência e motivo da institucionalização, $49 \%$ dos idosos eram provenientes de domicílio próprio e $30 \%$ moravam sozinhos e tinham dificuldades para realizar AVDs, sendo este o motivo principal da institucionalização.

O tempo médio de institucionalização dos idosos foi de 70 meses, variando entre 1 e 328 meses. Observou-se que $10,3 \%$ deles eram tabagistas, $61,9 \%$ apresentavam hipertensão arterial e $19,6 \%$ diabetes mellitus. A maioria $(72,2 \%)$ foi diagnosticada com doenças cardiovasculares, 35,1\% com doenças neurológicas e 30,9\% com doenças psiquiátricas.

A maioria dos idosos que compuseram a amostra $(80,4 \%)$ faziam uso de neurolépticos ou psicotrópicos e 57,7\% faziam uso de medicamentos anti-hipertensivos. Apenas 2,1\% dos idosos não faziam uso de nenhum tipo de medicamento. 
Tabela 1 - Distribuição dos idosos institucionalizados de Itaúna-MG, em 2009, segundo dados sócio-demográficos.

\begin{tabular}{|c|c|c|}
\hline & \multicolumn{2}{|c|}{ Total $(n=97)$} \\
\hline & $\mathrm{N}$ & $\%$ \\
\hline \multicolumn{3}{|l|}{ Sexo } \\
\hline Masculino & 40 & 41 \\
\hline Feminino & 57 & 59 \\
\hline \multicolumn{3}{|l|}{ Categorização da Idade } \\
\hline $60-64$ anos a & 6 & 6 \\
\hline $65-69$ anos & 14 & 14 \\
\hline $70-74$ anos & 22 & 23 \\
\hline $75-79$ anos & 16 & 16 \\
\hline 80 e mais & 39 & 40 \\
\hline \multicolumn{3}{|l|}{ Grau de Instrução } \\
\hline Analfabeto(a) & 53 & 55 \\
\hline Alfabetizado(a) & 39 & 40 \\
\hline Nível Fundamental & 1 & 1 \\
\hline Nível Médio & 3 & 3 \\
\hline Nível Superior & 0 & 0 \\
\hline Não Informado & 1 & 1 \\
\hline \multicolumn{3}{|l|}{ Cor ou Raça } \\
\hline Branca & 65 & 67 \\
\hline Preta & 15 & 15 \\
\hline Amarela / Indígena & 0 & 0 \\
\hline Parda & 17 & 18 \\
\hline \multicolumn{3}{|l|}{ Estado Civil } \\
\hline Solteiro(a) & 61 & 63 \\
\hline Casado(a) & 3 & 3 \\
\hline Viúvo(a) & 22 & 23 \\
\hline Amasiado(a) & 0 & 0 \\
\hline Divorciado(a) & 11 & 11 \\
\hline \multicolumn{3}{|l|}{ Número de Filhos } \\
\hline Média & 1 & \\
\hline Mínimo & 0 & \\
\hline Máximo & 9 & \\
\hline Desvio Padrão & 1,9 & \\
\hline \multicolumn{3}{|l|}{ Local de Procedência } \\
\hline Domicílio Próprio & 48 & 49 \\
\hline Domicílio de Parentes & 25 & 26 \\
\hline ILPI/Casa para Idosos & 11 & 11 \\
\hline Desabrigado & 4 & 4 \\
\hline Hospital & 3 & 3 \\
\hline Não Informado & 6 & 6 \\
\hline \multicolumn{3}{|l|}{ Motivo Principal de Institucionalização } \\
\hline Morava Sozinho/ Dificuldades para Realizar AVD & 29 & 30 \\
\hline Dificuldade Financeira & 3 & 3 \\
\hline Sofria Maus Tratos & 3 & 3 \\
\hline Opção & 12 & 12 \\
\hline Morava com Outro Idoso & 7 & 7 \\
\hline Dificuldade da Família em Prestar Cuidados/ Relacionamento & 25 & 26 \\
\hline Abandono & 3 & 3 \\
\hline Sem Informação & 15 & 15 \\
\hline \multicolumn{3}{|l|}{ Tempo de Institucionalização (em meses) } \\
\hline Média & 70 & \\
\hline Mínimo & 1 & \\
\hline Máximo & 328 & \\
\hline Desvio Padrão & 74 & \\
\hline
\end{tabular}


Tabela 2 - Distribuição dos idosos institucionalizados de Itaúna-MG, em 2009, segundo o nível de independência funcional pelo Índice de Katz.

\begin{tabular}{|c|c|c|}
\hline \multirow{2}{*}{ Índice de Katz (IK) $)^{(11)}$} & \multicolumn{2}{|c|}{ Total $(n=97)$} \\
\hline & $\mathrm{N}$ & $\%$ \\
\hline 0 - Independente em todas as funções & 22 & 23 \\
\hline 1 - Independente em cinco funções e dependente em uma função & 13 & 13 \\
\hline 2 - Independente em quatro funções e dependente em duas funções & 10 & 10 \\
\hline 3 - Independente em três funções e dependente em três funções & 5 & 5 \\
\hline 4 - Independente em duas funções e dependente em quatro funções & 16 & 16 \\
\hline 5 - Independente em uma função e dependente em cinco funções & 19 & 20 \\
\hline 6 - Dependente em todas as funções & 12 & 12 \\
\hline
\end{tabular}

Ao determinar o nível de independência para a realização de atividades pertencentes às seis funções: banhar-se, vestir-se, ir ao banheiro, transferir-se da cama para a cadeira e vice-versa, ser continente e alimentar-se, observou-se que 65,5\% dos idosos eram dependentes para tomar banho, 51,7\% para vestir-se, 44,8\% para usar o vaso sanitário, 33,3\% para transferir-se, 55,2\% eram portadores de incontinência e 9,2\% eram dependentes para alimentar-se.

Em relação ao Índice de Katz (IK) $23 \%$ dos indivíduos da amostra eram independentes nas 6 funções $(\mathrm{IK}=0)$, 77\% possuíam alguma dependência para AVDs (IK=1, 2, 3, 4, 5 ou 6), sendo $12 \%$ dependentes em todas as funções (Tabela 2 ).

\section{DISCUSSÃO}

O processo acelerado de transição demográfica tem sido associado em diversos países ao aumento na demanda por ILPI e esses fatos têm sido também vivenciados atualmente no Brasil. A correlação multicausal entre a estrutura etária da população e a demanda por ILPI é determinada pelo seu perfil social e de saúde. Os principais fatores considerados predisponentes à institucionalização dos idosos são: doenças crônico-degenerativas e suas sequelas, hospitalização recente e dependência para realizar AVDs ${ }^{(13)}$.

Associada a esses fatores, verifica-se uma nova configuração familiar, em que a mulher está inserida no mercado de trabalho, não permanecendo mais tão disponível para a prestação de cuidados aos idosos de sua família. A falta de um cuidador, somada às dificuldades de ordem financeira da maioria das famílias brasileiras, tem gerado obstáculos para a manutenção do idoso em seu lar.

O fato de muitos idosos brasileiros necessitarem morar sozinhos, de não contarem com suporte social satisfatório, além de possuírem baixa renda pode levar ao aumento na demanda dessas pessoas por ILPI.

A maioria dos idosos institucionalizados da cidade do interior de Minas Gerais onde foi realizado este estudo era do sexo feminino, de raça branca, com média de idade em torno de 77 anos. Esses dados foram semelhantes aos obtidos no estudo conduzido com 94 idosos de quatro instituições de longa permanência do interior de Minas Gerais. A autora constatou um predomínio de mulheres $(62,8 \%)$, que pode ser justificado pela maior expectativa de vida entre elas, e da raça branca $(68,1 \%)$. Apurou-se ainda uma média de idade em torno de 79,1 anos entre aqueles idosos $( \pm 9,59)^{(14)}$.

Em estudo com o objetivo de descrever o perfil de 80 octogenários de uma cidade do interior de São Paulo-Brasil, observou-se que 58 eram mulheres idosas e 22 eram homens idosos $^{(15)}$. Em outro estudo brasileiro com o objetivo de identificar as características demográficas, socioeconômicas e a situação de saúde de 98 idosos da área de abrangência de um Programa de Saúde da Família (PSF), constatou-se um predomínio na população idosa feminina $(62,2 \%)$, com média de idade de 69,9 anos para as mulheres e 68,8 anos para os homens ${ }^{(16)}$. O número de mulheres idosas é maior que o de homens em todos os grupos etários e essa diferença torna-se ainda maior com o aumento da idade (75 anos e mais), em consequência das maiores taxas de mortalidade experimentadas pelos homens e à maior longevidade das mulheres ${ }^{(17)}$.

Tratando-se de escolaridade, observou-se que a maioria dos idosos era analfabeta, sendo que a porcentagem de muIheres analfabetas era maior que a de homens analfabetos. Os idosos apresentavam baixo nível de escolaridade, e esse declinava ainda mais com o aumento da idade, sendo essa situação mais frequente nas mulheres, quando comparadas com os homens ${ }^{(17)}$. O alto índice de analfabetismo presente na população idosa se deve, principalmente, às dificuldades de acesso à escola no passado ${ }^{(15,18)}$. A diferença entre os sexos reflete as menores oportunidades de acesso à escola sofridas pelas mulheres ${ }^{(17-18)}$, atribuídas a uma cultura que não valorizava a educação escolar para as mulheres, que muitas vezes eram criadas para serem boas esposas, mães e donas-de-casa(15).

A maioria dos idosos institucionalizados que compuseram a amostra era solteira, sem filhos, provenientes de domicílio 
próprio, onde moravam sozinhos e tinham dificuldades para realizar AVDs. O tempo médio de institucionalização era de 70 meses, variando entre 1 e 328 meses. Pelo fato de não terem condições físicas e até mesmo psicossociais para viverem sozinhos, e por não terem suporte familiar de filhos ou cônjuges, muitos se institucionalizaram. Os novos arranjos familiares, como aqueles em que a mulher mora só, são mães solteiras ou casais sem filhos e filhos que emigraram, reduzem a perspectiva de envelhecimento com um suporte familiar, aumentando o risco para a institucionalização ${ }^{(13)}$. Depois que os idosos vão viver em ILPI, por lá permanecem por muito tempo ou até a morte. Como a institucionalização, geralmente, é a última opção para os idosos, a possibilidade de voltarem a viver na comunidade ou com a família é muito pequena.

A maioria $(72,2 \%)$ dos idosos institucionalizados apresentava doenças cardiovasculares, 35,1\% doenças neurológicas e 30,9\% doenças psiquiátricas. Observou-se que grande número de idosos $(80,4 \%)$ fazia uso de neurolépticos ou psicotrópicos e que $57,7 \%$ faziam uso de anti-hipertensivos. Estes resultados corroboram os resultados de estudo realizado em quatro instituições de longa permanência para idosos do interior de Minas Gerais, em que 52,1\% dos idosos institucionalizados usavam neurolépticos ou psicotrópicos e 38,3\% anti-hipertensivos $^{(14)}$.

Ao se avaliar o nível de independência para realização de AVDs, observou-se que muitos dos idosos (77\%) institucionalizados possuíam alguma dependência para AVDs, sendo $23 \%$ deles independentes. Em estudo com o objetivo de identificar o grau de independência para a realização de AVDs dos idosos residentes nas instituições da cidade de Taubaté-SP, verificou-se que $37 \%$ dos idosos institucionalizados podiam ser considerados independentes para o desempenho das AVDs, segundo o Índice de Katz ${ }^{(12)}$. Nas ILPI de Belo Horizonte, parcela significativa dos idosos institucionalizados era de idosas independentes e homens com menos de 65 anos. A maioria destas instituições não aceitavam idosos portadores de alguma demência ou acamados e várias recusavam idosos diagnosticados com doenças orgânicas ${ }^{(12)}$. As instituições locais onde este estudo foi realizado não apresentavam estes tipos de exigência para receber os idosos que muitas vezes eram recebidos já nestas condições, o que poderia justificar uma prevalência maior de idosos dependentes nestas ILPI.

Estudo realizado em Belo Horizonte evidenciou que, entre os idosos recenseados, os que se encontravam fragilizados representavam uma pequena parcela da população com 60 anos ou mais. Assim, devem ser propostos programas de prevenção de agravos e ações de promoção da saúde para promover a manutenção da capacidade funcional do idoso. Ao manter habilidades físicas e mentais necessárias a uma vida de forma independente e autônoma, os idosos poderão desfrutar de uma vida com maior qualidade no seu lar, junto de seus familiares, evitando ou postergando uma institucionalização ${ }^{(5)}$.

No Estatuto do Idoso, sancionado em agosto de 2003 e destinado a regular os direitos assegurados às pessoas com idade igual ou superior a 60 anos, consta que os idosos gozam de todos os direitos fundamentais inerentes à pessoa humana, sendo asseguradas a eles, por lei ou por outros meios, facilidades para a preservação de sua saúde física e mental, seu aperfeiçoamento moral, intelectual, espiritual e social em condições de liberdade e dignidade. Consta também que é obrigação da família, comunidade, sociedade e do Poder Público assegurar ao idoso, com absoluta prioridade, a efetivação do direito à vida, saúde, alimentação, educação, cultura, esporte, lazer, trabalho, cidadania, liberdade, dignidade, respeito e convivência familiar e comunitária(7).

\section{CONSIDERAÇÕES FINAIS}

O envelhecimento populacional tem trazido preocupações para os profissionais enfermeiros com relação ao planejamento e à implementação de cuidados que visem melhorar a qualidade de vida dos idosos. Esta preocupação tem sido um incentivo para a realização de estudos que possam melhor caracterizar este grupo e discutir intervenções de enfermagem que mais se apliquem às situações clínicas e problemas de vida desta população.

Os idosos que estão institucionalizados despertam uma preocupação ainda maior pela fragilidade social, psicológica e física em que podem se encontrar. Percebe-se que estes idosos demandam, progressivamente, cuidados de enfermagem mais qualificados, justificados pelo nível de dependência, patologias instaladas, número de medicamentos utilizados e riscos de complicações apresentadas. Por outro lado, muitas ILPI não estão preparadas para oferecer um cuidado adequado, sobretudo no que diz respeito à estrutura física e melhor qualificação do pessoal que presta os cuidados, muitas vezes leigos e despreparados.

Segundo os resultados obtidos, observou-se predomínio de idosos do sexo feminino, com média de idade 77 anos, analfabetos, brancos, solteiros e sem filhos. A maioria era proveniente de domicílio próprio, sendo que muitos idosos moravam sozinhos e tinham dificuldades para realizar AVDs, motivo principal da institucionalização. O tempo médio de institucionalização foi de 70 meses. O tabagismo esteve presente entre os idosos que apresentavam doenças cardiovasculares, neurológicas e psiquiátricas. Houve predomínio de uso de neurolépticos/psicotrópicos e anti-hipertensivos pelos idosos. Verificou-se que grande parte dos idosos institucionalizados encontrava-se em situação de dependência para o desempenho de AVDs e requerendo cuidados pessoais em tempo integral.

Para o incremento na qualidade de vida de idosos institucionalizados acredita-se que diversos fatores devem ser monitorados, entre eles a capacidade funcional, na maioria das vezes deficiente. No entanto, para oferecer uma atenção adequada, segura, ética e com qualidade ao idoso, faz-se necessária a efetivação de políticas públicas que atendam às reais necessidades desta população, além da capacitação dos profissionais de enfermagem para o cuidado do idoso institucionalizado. Dessa forma, os profissionais responsáveis pela assistência prestada a essa população devem estar orientados sobre a importância do cuidado integral e interdisciplinar, a fim contribuir para a melhoria na qualidade de vida dos idosos naquelas comunidades. 


\section{REFERÊNCIAS}

1. Scharfstein EA. Instituições de Longa Permanência: uma alternativa de moradia para idosos brasileiros na vida contemporânea. Rio de Janeiro. Tese [Doutorado em Psicologia]- Universidade Federal do Rio de Janeiro; 2006.

2. Instituto Brasileiro de Geografia e Estatística [homepage na internet]. Perfil dos Idosos responsáveis pelos domicílios. [acesso em 26 jul 2007] Disponível em: < http:// www.ibge.gov.br $>$.

3. Instituto Brasileiro de Geografia e Estatística [homepage na internet]. Censo Demográfico 2010. 2002. [acesso em 05 jan 2011] Disponível em: <http://www.ibge.gov.br>.

4. Matos AB. Itaúna em dados: ano 2010 - ano base 2009. 5. ed. Itaúna; 2010.

5. Tannure MC, Alves M, Sena RR, Chianca TCM. Perfil epidemiológico da população idosa de Belo Horizonte-MG, Brasil. Rev Bras Enferm. [periódico na Internet]. 2010 [acesso em 03 jan 2011];63(5): 817-22. Disponível em: < http://www.scielo.br/pdf/reben/v63n5/20.pdf>.

6. Ministério da Saúde (Brasil) Secretaria de Atenção à Saúde. Departamento de Atenção Básica. Envelhecimento e Saúde da Pessoa Idosa. Caderno de Atenção Básica. Brasília: Ministério da Saúde 2007. (Normas e manuais técnicos $-\mathrm{n} .^{\circ} 19$ ).

7. Ministério da Saúde (Brasil) Estatuto do Idoso. 2. ed. Brasília; 2006.

8. Costa EFA, Monego ET. Avaliação Geriátrica Ampla (AGA). Rev UFG [periódico na Internet]. 2003 [acesso em 28 jul 2007];5(2). Disponível em <http://www. proec. ufg.br $>$.

9. Minas Gerais. Secretaria de Estado de Saúde. Atenção à saúde do idoso. Belo Horizonte: SAS/MG; 2006.

10. Duarte $\mathrm{YAO}$, Andrade CL, Lebrão ML. O Índex de Katz na avaliação da funcionalidade dos idosos. Rev Esc Enferm
USP 2007;41(2):317-25.

11. Lino VTS, Pereira SRM, Camacho LAB, Filho STR, Buksman S. Adaptação transcultural da Escala de Independência em Atividades da Vida Diária (Escala de Katz). Cad Saúde Pública 2008;24(1):103-12.

12. Araújo MOPH, Ceolim MF. Avaliação do grau de independência de idosos residentes em instituições de longa permanência. Rev Esc Enferm USP 2007;41(3):378-85.

13. Chaimowickz F, Greco DB. Dinâmica da institucionalização de idosos em Belo Horizonte, Brasil. Rev Saúde Pública 1999;33(5):454-60.

14. Souza DMST. Incidência de úlceras por pressão e fatores de risco em idosos institucionalizados São Paulo. Dissertação [Mestrado em Enfermagem]- Universidade de São Paulo; 2005.

15. Inouye K, Pedrazzani ES. Nível de instrução socioeconômico e avaliação de algumas dimensões da qualidade de vida de octogenários. Rev Latino-am. Enferm [periódico na Internet]. 2007 [citado 2009 out 09];15(n. ${ }^{\circ}$ esp) Disponível em: < http://www.revistasusp.sibi.usp.br/pdf/rsp/ v41n5/5764.pdf $>$.

16. Souza LM, Morais EP, Barth QCM. Características demográficas, socioeconômicas e situação de saúde de idosos de um programa de saúde da família de Porto Alegre, Brasil. Rev Latino-am. Enferm 2006;14 (6):79-85.

17. Alves LC. Perfil demográfico, socioeconômico e de condições de saúde dos idosos do município de São Paulo, 1999/2000. In: XIV Encontro Nacional de Estudos Populacionais; 2004 set 20-24; Caxambu (MG), Brasil. Caxambu: ABEP; 2004. p.17.

18. Paskulin LMG, Vianna LAC. Perfil sociodemográfico e condições de saúde auto-referidas de idosos de Porto Alegre. Rev Saúde Pública 2007;41(5):757-68. 